\title{
STABILITY RESULTS FOR PERIODIC SECOND ORDER LINEAR DIFFERENTIAL EQUATIONS
}

\author{
L. H. ERBE ${ }^{1}$
}

\begin{abstract}
Sufficient conditions for stability, asymptotic stability and instability of the trivial solution of a periodic second order linear differential equation are obtained.
\end{abstract}

1. Introduction. Consider the second order linear differential equation

$$
L x=x^{\prime \prime}+p_{1}(t) x^{\prime}+p_{0}(t) x=0,
$$

where $p_{i}(t)$ are continuous real-valued functions of period $\omega$, and $\omega$ is a positive constant. In this note we obtain sufficient conditions for stability, asymptotic stability and instability of the trivial solution of (1.1). These properties are, of course, completely determined by the Floquet characteristic multipliers $\lambda_{1}, \lambda_{2}$ of $L x=0$; i.e., the roots of the equation

$$
\lambda^{2}-A \lambda+B=0,
$$

where $\lambda_{1} \lambda_{2}=B=\exp (-\omega J), J=(1 / \omega) \int_{0}^{\omega} p_{1}(t) d t$ and $A=x_{1}(\omega)+x_{2}^{\prime}(\omega)$. Here $x_{1}, x_{2}$ are solutions of $L x=0$ satisfying $x_{1}(0)=1, x_{1}^{\prime}(0)=0, x_{2}(0)=0, x_{2}^{\prime}(0)=1$. Therefore, there exists no simple method for explicitly determining $\lambda_{1}, \lambda_{2}$, given $p_{i}(t)$. However, the stability properties of $L x=0$ may be related to periodic boundary value problems and it is this method which we shall employ here. We refer to the book of Hartman [3] and the monograph of Magnus and Winkler [6] for a discussion of Floquet theory; in particular, the latter deals with the second order equation in the form of Hill's equation. The papers [1, 2,5 and 9] have discussed the stability problem for the third order case.

It is natural to consider three cases when discussing the stability of (1.1) according as $J$ is positive, zero, or negative. Thus, a necessary condition for asymptotic stability of (1.1) is $J>0$ since $\lambda_{1} \lambda_{2}=B<1$. Similarly, stability occurs only if $J=0$ (i.e., all solutions are bounded for all $t$ ). Likewise, there will exist linearly independent unbounded solutions of Floquet type only if $J<0$.

We shall consider the characteristic polynomial

$$
Q(\gamma, t)=\gamma^{2}+p_{1}(t) \gamma+p_{0}(t)
$$

Received by the editors September 8, 1983 and, in revised form, January 31, 1984.

1980 Mathematics Subject Classification. Primary 34B30, 34C11, 34C25, 34D05.

Key words and phrases. Periodicity, Floquet theory, stability.

${ }^{1}$ Research supported by NSERC Grant A-7673 and the Alexander von Humboldt Foundation.

(C)1985 American Mathematical Society $0002-9939 / 85 \$ 1.00+\$ .25$ per page 
and we define the sets $S, T$ of real numbers by

$$
\begin{aligned}
& S=\{\gamma: Q(\delta, t) \geqslant 0 \text { for all } \delta \leqslant \gamma, 0 \leqslant t \leqslant \omega\}, \\
& T=\{\gamma: Q(\delta, t) \geqslant 0 \text { for all } \delta \geqslant \gamma, 0 \leqslant t \leqslant \omega\} .
\end{aligned}
$$

We note that $S$ is not bounded above iff $T$ is not bounded below iff $S=T=$ $(-\infty,+\infty)$. If $S$ is bounded above then we set

$$
\alpha=\sup S, \quad \beta=\inf T
$$

so that $\alpha \leqslant \beta$. In fact, since $S$ is bounded above iff $Q\left(\gamma_{0}, t_{0}\right)<0$ for some $\left(\gamma_{0}, t_{0}\right)$, it follows by continuity that $Q\left(\gamma, t_{0}\right)<0$ for all $\gamma$ near $\gamma_{0}$, and hence $\alpha<\beta$. In $\S 2$ we shall obtain bounds on the characteristic multipliers $\lambda_{1}, \lambda_{2}$ in terms of $\alpha, \beta$, and shall discuss the application of these techniques to some examples in $\S 3$. These lead to some bounds for the multipliers of Hill's equation ( $\$ 3$, Example 2). The results obtained apply to parameter values in the so-called zeroth interval or zone of instability. We do not, however, discuss intervals of stability and instability for Hill's equation. The monograph [6] contains an excellent discussion along with additional pertinent references.

2. Recall that an $n$th order linear differential equation is said to be disconjugate on an interval $I$ in case no nontrivial solution has more than $n-1$ zeros (counting multiplicities) on $I$. We begin with a result which gives upper and/or lower bounds on the real characteristic multipliers of (1.1).

THEOREM 2.1. Assume that $L x=0$ is disconjugate on $[\tau, \tau+\omega]$ for all $0 \leqslant \tau<\omega$. Assume further that for any $\gamma \in S(\gamma \in T)$ we have $Q(\gamma, t) \not \equiv 0$ on $[0, \omega]$. Then:

(a) if $S$ is bounded above, we have

$$
e^{\alpha \omega}<\lambda \quad\left(\lambda<e^{\beta \omega}\right),
$$

for all characteristic multipliers $\lambda$ of (1.1);

(b) if $S=(-\infty,+\infty)=T$, then (1.1) has only complex characteristic multipliers.

Proof. Suppose first that $S$ is bounded above so that $\alpha<\beta$ are well defined by (1.6). If there exists a negative characteristic multiplier, then there exists a solution $v(t)$ (nontrivial) of (1.1) with $v(t+\omega)=\mu v(t)$ for some $\mu<0$. But then there exists $\tau \in[0, \omega]$ with $v(\tau)=0$ and hence $v(\tau+\omega)=0$, a contradiction. Therefore, all real characteristic multipliers are positive. Suppose next that $\lambda \geqslant e^{\beta \omega}$ is a multiplier. Then by Floquet theory, there exists a solution $w(t)$ (nontrivial) of (1.1) which can be written in the form $w(t)=q(t) e^{\gamma t}$, where $\gamma=(1 / \omega) \ln \lambda \geqslant \beta$ and $q(t+\omega)=$ $q(t)$ is continuous. Since $(1.1)$ is disconjugate on $[\tau, \tau+\omega]$ for all $0 \leqslant \tau<\omega$, it follows that $q(t)>0$ on $[0, \omega]$. Let $u(t) \equiv e^{\gamma t}(M q(t)-1)$, where $M=\max (1 / q(t))$ $=1 / q\left(t_{0}\right)$. Then $u\left(t_{0}\right)=u\left(t_{0}+\omega\right)=0$ and $u(t) \geqslant 0$ on $\left[t_{0}, t_{0}+\omega\right]$. Also,

$$
L u(t)=-Q(\gamma, t) \equiv g(t) \leqslant 0 \text { on }\left[t_{0}, t_{0}+\omega\right] .
$$

On the other hand, the solution to the BVP

$$
L x=g(t), \quad x\left(t_{0}\right)=x\left(t_{0}+\omega\right)=0
$$

may be written as

$$
u(t)=\int_{t_{0}}^{t_{0}+\omega} G(t, s) g(s) d s,
$$


where $G(t, s)$ is the Green's function for (2.3). Since $L x=0$ is disconjugate, $G(t, s) \geqslant 0$ for $t, s \in\left[t_{0}, t_{0}+\omega\right]$. Therefore, (2.4) shows that $u(t) \leqslant 0$ on $\left[t_{0}, t_{0}+\omega\right]$ and hence $u(t) \equiv 0$ so that $q(t) \equiv$ const. But then $Q(\gamma, t) \equiv 0$ on $\left[t_{0}, t_{0}+\omega\right]$, a contradiction. Therefore, we must have $\lambda<e^{\beta \omega}$. Similarly, one shows that $\lambda>e^{\alpha \omega}$ must hold. This proves part (a).

(b) If $S=(-\infty,+\infty)$, then, as in the proof of part (a), there are no negative characteristic multipliers and the assumption that $Q(\gamma, t) \geqslant 0$ and $\neq \equiv 0$ on $[0, \omega]$ for all $\gamma \in(-\infty,+\infty)$ guarantees that there are also no positive characteristic multipliers. This proves part (b).

For convenience, we list several conditions which will be needed subsequently.

$\left(\mathrm{H}_{1}\right) \beta \leqslant 0$ and for any $\gamma \in T, Q(\gamma, t) \not \equiv 0$ on $[0, \omega]$.

$\left(\mathrm{H}_{2}\right) \alpha \geqslant 0$ and for any $\gamma \in S, Q(\gamma, t) \not \equiv 0$ on $[0, \omega]$.

$\left(\mathrm{H}_{3}\right) \beta \leqslant-\frac{1}{2} J$ and for any $\gamma \in T, Q(\gamma, t) \neq 0$ on $[0, \omega]$.

$\left(\mathrm{H}_{4}\right) \alpha \geqslant-\frac{1}{2} J$ and for any $\gamma \in S, Q(\gamma, t) \neq 0$ on $[0, \omega]$.

We may now prove

TheOREM 2.2. Assume $J>0$ and that $L x=0$ is disconjugate on $[\tau, \tau+\omega]$ for all $0 \leqslant \tau<\omega$. Further, let one of the conditions $\left(\mathrm{H}_{1}\right),\left(\mathrm{H}_{3}\right)$, or $\left(\mathrm{H}_{4}\right)$ hold. Then the BVP

$$
L x=f(t), \quad x(0)=x(\omega) \quad(f(t+\omega)=f(t))
$$

has a unique asymptotically stable periodic solution for any periodic continuous $f$. Further, if $\left(\mathrm{H}_{3}\right)$ or $\left(\mathrm{H}_{4}\right)$ holds, then all characteristic multipliers of (1.1) are complex so that $L x=0$ is oscillatory on $(-\infty,+\infty)$.

Proof. It suffices to show that any one of the conditions $\left(\mathrm{H}_{1}\right), \cdot\left(\mathrm{H}_{3}\right)$, or $\left(\mathrm{H}_{4}\right)$ implies that the characteristic multipliers $\lambda_{1}, \lambda_{2}$ of (1.1) satisfy $\left|\lambda_{i}\right|<1, i=1,2$. Since $B=\lambda_{1} \lambda_{2}<1$, we see that if $\left(\mathrm{H}_{1}\right)$ holds, then there are no real multipliers $\geqslant 1$ by Theorem 2.1. Since the complex multipliers occur in conjugate pairs, it follows that $\left|\lambda_{i}\right|<1, i=1,2$. If $\left(\mathrm{H}_{3}\right)$ holds, then any real characteristic multiplier satisfies $0<\lambda<e^{\beta \omega}$. Thus, if both $\lambda_{1}, \lambda_{2}$ are real, then we would have $\lambda_{1} \lambda_{2}<e^{2 \beta \omega} \leqslant e^{-\omega J}$ $=B=\lambda_{1} \lambda_{2}$, a contradiction. Therefore, both $\lambda_{1}, \lambda_{2}$ are complex and $\left|\lambda_{1}\right|=\left|\lambda_{2}\right|<1$. Similarly, if $\left(\mathrm{H}_{4}\right)$ holds and $\lambda_{1}, \lambda_{2}$ are both real, we would have $B=\lambda_{1} \lambda_{2}>e^{2 \alpha \omega} \geqslant$ $e^{-\omega J}=B$, again a contradiction. Thus, either $\left(\mathrm{H}_{3}\right)$ or $\left(\mathrm{H}_{4}\right)$ implies that $\lambda_{1}, \lambda_{2}$ are complex and hence the corresponding solutions are oscillatory. This completes the proof.

Similarly, we may establish the following two corollaries for the cases $J=0$ and $J<0$, respectively.

Corollary 2.3. Assume $J=0$ and $L x=0$ is disconjugate on $[\tau, \tau+\omega]$ for all $0 \leqslant \tau<\omega$, and let condition $\left(\mathrm{H}_{1}\right)$ or $\left(\mathrm{H}_{2}\right)$ hold. Then the BVP (2.5) has a unique stable periodic solution. That is, the characteristic multipliers $\lambda_{1}, \lambda_{2}$ of (1.1) are complex and satisfy $\left|\lambda_{1}\right|=\left|\lambda_{2}\right|=1$, and $L x=0$ is oscillatory.

PROOF. Since $B=\lambda_{1} \lambda_{2}=1$ and since either $\left(\mathrm{H}_{1}\right)$ or $\left(\mathrm{H}_{2}\right)$ implies that there are no real characteristic multipliers $\geqslant 1$ or $\leqslant 1$, respectively, the result follows.

The following result may be obtained in a manner similar to Theorem 2.2 and Corollary 2.3 so we omit the proof. 
Corollary 2.4. Assume $J<0$ and $L x=0$ is disconjugate on $[\tau, \tau+\omega]$ for all $0 \leqslant \tau<\omega$, and let one of the conditions $\left(\mathrm{H}_{2}\right),\left(\mathrm{H}_{3}\right)$, or $\left(\mathrm{H}_{4}\right)$ hold. Then the BVP (2.5) has a unique unstable periodic solution. That is, the characteristic multipliers $\lambda_{1}, \lambda_{2}$ of (1.1) satisfy $\left|\lambda_{i}\right|>1, i=1,2$. Further, if $\left(\mathrm{H}_{3}\right)$ or $\left(\mathrm{H}_{4}\right)$ holds, then $\lambda_{1}, \lambda_{2}$ are complex and hence $L x=0$ is oscillatory.

3. Examples. (1) Suppose $4 p_{0}(t) \geqslant\left(p_{1}(t)\right)^{2}$ (where if $4 p_{0}-p_{1}^{2} \equiv 0$ we assume $p_{0} \not \equiv$ const.). Let $M=\max _{[0, \omega]}\left|p_{1}(t)\right|$ and assume $\int_{0}^{\omega} p_{0} d t+2 M \leqslant 4 / \omega$. It follows that $L x=0$ is disconjugate on $[\tau, \tau+\omega]$ for all $0 \leqslant \tau<\omega$ (cf. [3, Example 5.3, p. 346]). Since $Q(\gamma, t) \geqslant 0$ and $\not \equiv 0$ for all $\gamma \in(-\infty,+\infty)$, it follows from Theorem 2.1 that $L x=0$ has only complex characteristic multipliers. Therefore, $L x=0$ is oscillatory and is stable, asymptotically stable, or unstable according as $J=$ $(1 / \omega) \int_{0}^{\omega} p_{1} d t$ is zero, positive, or negative. If $p_{1} \equiv 0$, this reduces to an old result of Liapunov [4].

(2) In this example we consider an application to Hill's equation which we shall assume has the form

$$
x^{\prime \prime}+(-k+q(t)) x=0
$$

where $q(t)$ is periodic of period $\pi$ and $\int_{0}^{\pi} q(t) d t=0(q \not \equiv 0)$. It is well known (cf. $[6,7])$ that there exists a largest $k_{0}>0$ such that $(3.1)$ has a periodic nontrivial solution. If $k \geqslant k_{0}$, then all solutions are nonoscillatory and if $k<k_{0}$, all solutions are oscillatory (see e.g. [6, 7]). It is also well known that in the nonoscillatory case, a solution has at most one zero on $(-\infty, \infty)$ [7]. If we set $q_{1}=\min q(t)<0$, then for $k>k_{0}$ it follows that $\beta=\left(k-q_{1}\right)^{1 / 2}$ and $\alpha=-\beta$ so, by Theorem $2.1, \lambda_{1}, \lambda_{2}$ are real. If we assume for convenience that $\lambda_{1}>1$ so that $\lambda_{2}=1 / \lambda_{1}<1$, then we have

$$
1<\lambda_{1}<\exp \left(\pi\left(k-q_{1}\right)^{1 / 2}\right) .
$$

Magnus and Winkler in [6, Theorem 4.3] showed that (in our terminology) if $k>k_{0}$ and if $x(t)=p(t) e^{\mu t}$ is a Floquet type solution with $\mu>0, p(t+\pi)=p(t), p \neq 0$, then

$$
\mu^{2} \geqslant k-k_{0} \text {. }
$$

Since $\mu=1 / \pi \ln \lambda_{1},-\mu=1 / \pi \ln \lambda_{2}$, we see that (3.2) and (3.3) together yield the estimate

$$
k-k_{0} \leqslant \mu^{2}<k-q_{0}
$$

we remark also that Moore in [7] studied the two-parameter version of (3.1), $x^{\prime \prime}+(-k+m q(t))=0$, and obtained results concerning the region in the $k m$-plane for which the equation is nonoscillatory. Lower and upper bounds on the characteristic multipliers for this equation can be obtained in a manner similar to the above.

(3) Assume $J=(1 / \omega) \int_{0}^{\omega} p_{1} d t>0$. If there exists $c>0$ such that $c^{2}+p_{0}(t) \leqslant$ $c p_{1}(t)$ for all $0 \leqslant t \leqslant \omega$ and $p_{0}(t) \geqslant 0(\not \equiv 0), p_{1}(t) \geqslant 0$, then $\beta \leqslant 0$ and $L x=0$ is disconjugate on $(-\infty,+\infty)$ (i.e., the function $r \equiv-c$ is a solution of the Riccati differential inequality $r^{\prime}+r^{2}+p_{1} r+p_{0} \leqslant 0$ on $(-\infty,+\infty)$ (cf. [3, Theorem 7.2, p. 362])). Thus, it follows that $L x=0$ is asymptotically stable. 
(4) Let $a, b, c$ be real constants with $c>1, a, b>0$, and let $p_{0}(t) \equiv a(1+\sin 2 t)$, $p_{1}(t) \equiv b(c+\sin 2 t)$ so that $p_{0}(t) \geqslant 0$ and $J=(1 / \pi) \int_{0}^{\pi} p_{1}(t) d t=b c>0$. Also, since $\max \left|p_{0}(t)\right|=2 a, \max \left|p_{1}(t)\right|=b(c+1)$, we have by a result of Opial [8] (cf. [3, p. 346]) that $L x=0$ is disconjugate on $[t, t+\pi]$ for all $0 \leqslant t<\pi$ provided

$$
4 b(c+1)+8 a \pi<\pi \text {. }
$$

Thus, if (3.5) holds, then $Q(\gamma, t)=\gamma^{2}+p_{1}(t) \gamma+p_{0}(t) \geqslant 0$ for all $\gamma \geqslant 0$ so that $\beta \leqslant 0$. Moreover, $Q(\gamma, t) \geqslant 0$ if $\gamma<0$ and

$$
\gamma<b(1-c) \text {. }
$$

Therefore, $\alpha \geqslant b(1-c)$. We see then that if (3.5) holds, it follows that $L x=0$ is asymptotically stable and any real characteristic multiplier $\lambda$ satisfies

$$
\exp (b \pi(1-c))<\lambda<1 .
$$

Finally, if $c \leqslant 2$, then a calculation shows that $\alpha \geqslant-\frac{1}{2} b c=-\frac{1}{2} J$ and hence $L x=0$ is oscillatory by Theorem 2.2 (i.e., the characteristic multipliers $\lambda_{1}, \lambda_{2}$ are complex with $\left|\lambda_{i}\right|=B^{1 / 2}, i=1,2$ ).

Similar examples may also be given for the case $J<0$. We leave this to the interested reader.

\section{REFERENCES}

1. L. H. Erbe, Third order linear differential equations with periodic coefficients, Proc. Amer. Math. Soc. 64 (1977), 241-247.

2. Stability and periodicity for linear differential equations with periodic coefficients, Ann. Polon. Math. 31 (1975), 131-140.

3. P. Hartman, Ordinary differential equations, Wiley, New York, 1964.

4. A. Liapunov, Problème général de la stabilité du movement, Ann. Fac. Sci. Univ. Toulouse 9 (1907), 203-475 (reproduced in Ann. of Math. Studies, vol. 17, Univ. Press, Princeton, N.J., 1947).

5. G. P. Loś, $A$ sufficient test for the stability of the trivial solution of a third order differential equation with periodic coefficients, Differentsial'nye Uravneniya 3 (1967), 1707-1717. (Russian)

6. W. Magnus and S. Winkler, Hill's equation, Interscience, New York, 1966.

7. R. A. Moore, The least eigenvalue of Hill's equation, J. Analyse Math. 5 (1956-1957), 183-196.

8. Z. Opial, Sur une inegalité de C. de la Valleé Poussin dans la théorie de l'équation différentielle linéaire du second ordre, Ann. Polon. Math. 6 (1959), 87-91.

9. E. L. Tonkov and G. I. Yutkin, Periodic solutions and stability of a linear differential equation with periodic coefficients, Differentsial'nye Uravneniya 5 (1969), 1990-2001; English transl., Differential Equations 5 (1969), 1484-1492.

Department of Mathematics, University of Alberta, Edmonton T6G 2H1, Alberta, Canada 